\title{
Are Mauritia flexuosa L. f. palm swamps in the Brazilian Pantanal true veredas? A floristic appraisal
}

\section{Os buritizais (Mauritia flexuosa L. f.) no Pantanal brasileiro são verdadeiras veredas? Uma avaliação florística}

\author{
Suzana Neves Moreiral', Vali Joana Pott", Evaldo Benedito de Souza", Arnildo Pott" \\ Universidade Estadual de Mato Grosso do Sul. Coxim, Mato Grosso do Sul, Brasil \\ "Universidade Federal de Mato Grosso do Sul. Campo Grande, Mato Grosso do Sul, Brasil
}

\begin{abstract}
In this first report on the flora of Mauritia flexuosa L. f. palm swamps in the Pantanal wetland of Brazil, we surveyed and compared the angiosperm flora of eight areas of palm swamps in the Pantanal plain to determine if they correspond to veredas. Studied areas were four sites near the upland in Rio Verde and Coxim, and four in the Parque Estadual do Pantanal do Rio Negro, Aquidauana, state of Mato Grosso do Sul, Brazil. To determine species similarity, we compared our results with inventories of aquatic plants of the Pantanal and of species of veredas in the state. Using the wandering method, we recorded a total of 385 species of phanerogams. Species richness was higher in Rio Verde/Coxim (273 species) than in Rio Negro (243 species), with a similarity of $33.7 \%$. We found a rare and endemic plant, Polygala grazielae Marques, first record in the Pantanal. We found that both sites correspond to two types of palm swamps but with different floras. The species associated with $M$. flexuosa in the park are much the same as those of seasonally flooding grasslands of the Pantanal, while on the edge of the plain many species are common to the upland true veredas, particularly on organic soil. Thus, we conclude that the Rio Verde/Coxim areas are true veredas. These palm swamps and veredas in the Pantanal should receive the same legal protection as the veredas in Cerrado.
\end{abstract}

Keywords: Moriche palm. Flora. Grassland. Polygala. Savanna. Wetland.

Resumo: Este é o primeiro levantamento da flora dos buritizais (Mauritia flexuosa L. f.) no Pantanal. Pouco se sabe sobre a flora das veredas no Pantanal. Pesquisamos e comparamos a flora de angiospermas em oito formações com buriti na planície pantaneira, sendo quatro áreas próximas à área de planalto em rio Verde e Coxim, e quatro áreas no Parque Estadual do Pantanal do Rio Negro, em Aquidauana, estado de Mato Grosso do Sul, Brasil, através do método de caminhamento. Para determinar a similaridade de espécies, comparamos nossos resultados com os inventários das plantas aquáticas do Pantanal e das espécies das veredas do estado. Registramos um total de 385 espécies de fanerógamas. A riqueza de espécies foi maior no rio Verde/Coxim (273 espécies) do que em rio Negro (243), com uma similaridade de 33,7\%. Encontramos uma planta rara e endêmica, Polygala grazielae Marques, primeiro registro no Pantanal. Verificamos que os dois locais correspondem a dois tipos de buritizais, com diferentes floras. As espécies associadas a Mauritia flexuosa no rio Negro são praticamente as mesmas das áreas sazonalmente inundadas do Pantanal, enquanto na borda da planície (rio Verde/ Coxim) são mais similares às veredas verdadeiras do planalto, particularmente em solo orgânico. Assim, concluímos que as áreas de rio Verde/Coxim são verdadeiras veredas. Acreditamos que estas áreas com buritis e as veredas no Pantanal devem receber a mesma proteção que as veredas no Cerrado.

Palavras-chave: Buriti. Flora. Campo úmido. Polygala. Savana. Áreas úmidas.

MOREIRA, S. N., V. J. POTT, E. B. SOUZA \& A. POTT, 2017. Are Mauritia flexuosa L.f. palm swamps in the Brazilian Pantanal true veredas? A floristic appraisal. Boletim do Museu Paraense Emílio Goeldi. Ciências Naturais 12(2): 221-238.

Autora para correspondência: Suzana Neves Moreira. Universidade Estadual de Mato Grosso do Sul. Unidade Coxim. Rua General Mendes de Morais, 296. Coxim, MS, Brasil. CEP 79400-000 (suzannanevesmoreira@gmail.com).

Recebido em 22/04/2017

Aprovado em 21/09/2017

Responsabilidade editorial: Fernando da Silva Carvalho Filho

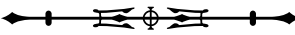




\section{INTRODUCTION}

Veredas correspond to one of the grassland elements in the Cerrado domain (Carvalho, 1991). They are environments with water table outcrop between gallery forests and the Cerrado proper, and they play an important link between the physiognomies (Oliveira-Filho \& Martins, 1986; Carvalho, 1991). Veredas are legally protected and considered Permanent Preservation Areas (Brasil, 2002). Nevertheless, these wetlands suffer anthropic impacts that can become irreversible (Oliveira et al., 2009), for being highly sensitive to disturbances and poorly resilient (Carvalho, 1991).

According to Conselho Nacional do Meio Ambiente (CONAMA) (Brasil, 2002), veredas are defined as swampy or waterlogged spaces, which contain springs or the headwaters of creeks, on hydromorphic soils, usually characterized by rows of moriche palms (Mauritia flexuosa) and other forms of typical vegetation. In an amendment to that definition, Moreira et al. (2015) carried out a wide sampling of the vegetation of wetlands with and without M. flexuosa and concluded that the moriche palm is only an additional species that permits the identification of a vereda, however, it is not an exclusive species to these environments.

The M. flexuosa palmland (buritizal) has been mapped in the Pantanal and is restricted to the eastern edge of the plain and corresponds to only $0.2 \%$ of the vegetation (Silva et al., 2000). There is an information gap on the floristics of the these palmlands or veredas in the Pantanal, except for brief reports on their flora, e.g., Pott et al. (2011), insufficient to provide a floristic inventory and to compare them with veredas and the aquatic macrophytes of Pantanal.

Therefore, we sought to inventory the flora of representative areas in order to compare the flora of two types of $M$. flexuosa swamps at eight sites in the Pantanal wetland in relation to the upland veredas and to the aquatic macrophytes of the Pantanal, to determine if the areas with moriche palms in the Pantanal can be considered veredas.

\section{MATERIAL AND METHODS}

We inventoried the angiosperm flora of eight selected areas of Mauritia flexuosa palm swamps in two sites in the Pantanal wetland. One sampled site was located in the Parque Estadual do Pantanal do Rio Negro, in the municipality of Aquidauana, and the other near the eastern border of the plain, in the municipalities of Rio Verde and Coxim, in Mato Grosso do Sul, Brazil (Figure 1), with four sample points per site. We used the wandering method (Filgueiras et al., 1994), whereby the area is inspected up and down a few times until no additional species are found. Sampling effort was similar between areas, with three botanists observing during c. 6 h per area of c. $100 \times 200$ m. Fertile specimens were collected for the CGMS and BHCB herbaria.

For a more accurate comparison between the targeted vegetation types, we compared three floristic lists. The first is the flora of veredas of Mato Grosso do Sul (see Moreira, 2015), the second one represents the object of the present work, and the third one shows the species of aquatic macrophytes of the Pantanal (see Pott et al., 2012). Based on the species occurrence, we calculated the Bray-Curtis similarity.

The eight sampling areas were the following:

- Site 1: Parque Estadual do Pantanal do Rio Negro, municipality of Aquidauana: riparian forest with $M$. flexuosa $19^{\circ} 44^{\prime} 17.1^{\prime \prime}$ S, $56^{\circ} 41^{\prime}$ 51' W, 100 m altitude; 19²9' $26^{\prime \prime} \mathrm{S}$, $56^{\circ} 33^{\prime} 22^{\prime \prime} \mathrm{W}, 104$ m altitude; swampy forest with $M$. flexuosa $19^{\circ} 44^{\prime} 25^{\prime \prime}$ S, 56 41' 47.5” W, 99 m altitude; floodable grassland with many dead and few young $M$. flexuosa $19^{\circ} 28^{\prime}$ 58.7" S, 56 41' 23.8” W, 98 m altitude; seasonal stream with M. flexuosa $19^{\circ} 29^{\prime} 44.8^{\prime \prime} \mathrm{S}, 56^{\circ} 40^{\prime} 27.8^{\prime \prime} \mathrm{W}$, 98 m altitude; $19^{\circ} 35^{\prime} 33^{\prime \prime} \mathrm{S}, 56^{\circ} 48^{\prime} 25^{\prime \prime} \mathrm{W}, 96$ m altitude;

- Site 2: municipality of Rio Verde: Ranch Fazenda Buritizal, stream Córrego Feioso 18 40’ 06.8” S, 5510' 59.1” W, 165 m altitude; stream Córrego Pindaivão, 18 49' 46.5" S, 55 07' 55.6" W, 187 m altitude; stream Córrego Pindaivão, $18^{\circ} 47^{\prime} 17.7^{\prime \prime}$ S, 55 04' 17.1" W, 176 m altitude; municipality of Coxim: ranch Fazenda Nova Esperança, 18 11' 05" S, 54 53' 23" W, 190 m altitude. 


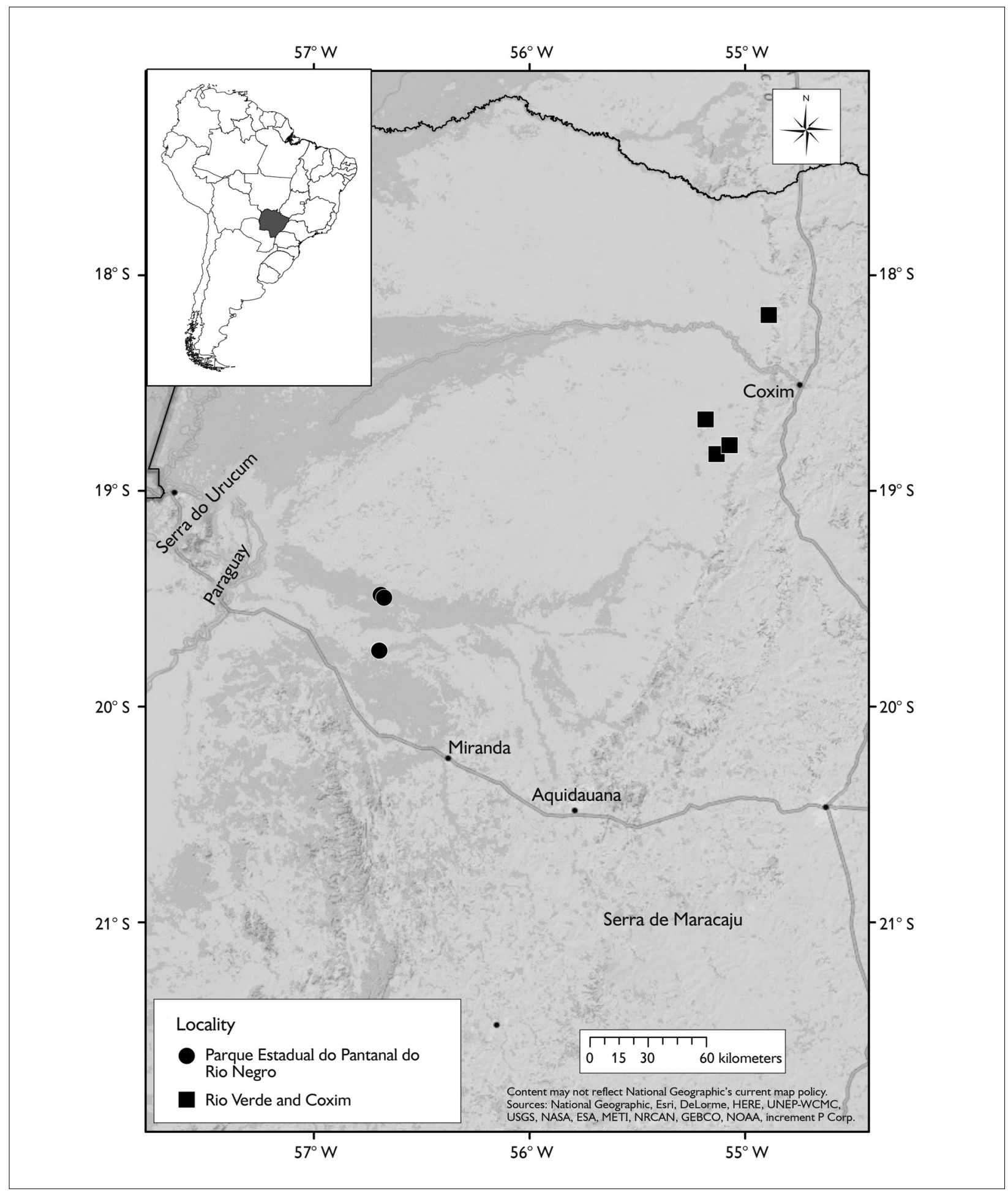

Figure 1. Map of the sampling areas in two sites, Parque Estadual do Pantanal do Rio Negro and Rio Verde/Coxim, in the Pantanal wetland, Mato Grosso do Sul, Brazil. 
The soils are sandy in the Rio Verde areas, but organic in Coxim, and sandy-loamy in the park. Flooding is short and shallow in the first, from local seasonal rain and water table rise forming streams, compared with the second site that is under influence of seasonal flooding by the Aquidauana River. The organic soil in the Coxim swamp is waterlogged nearly year round.

\section{RESULTS AND DISCUSSION}

We found 385 phanerogams in Mauritia flexuosa palm swamps in the Pantanal wetland, recording 243 species in the Rio Negro Park and 273 in Rio Verde/Coxim, both sites having 130 species in common (Appendix). Most species were collected elsewhere on the floodplain (Pott \& Pott, 2000). Comparing these results with the work reported by Moreira et al. (2015) in true veredas on the uplands, we can see that species sharing is on the order of only $30 \%$, showing more similarity with riparian and flooding grassland vegetation of the Pantanal wetland (Figure 2). Although the similarity dendrogram suggests the greatest sharing of species between the vegetation of the areas of $M$. flexuosa palmlands in the Pantanal and the aquatic vegetation of the Pantanal, we perceived that the similarity is low, approximately 40\% (Figure 2).

The studied M. flexuosa swamps on the lowland have a much lower species richness than the upland veredas, where over 1,000 species of phanerogams have been inventoried (Moreira, 2015). Obviously the sampling effort on the vegetation of veredas was much higher than on the vegetation composing the moriche palmlands in the Pantanal. Nevertheless, our preliminary results provide important information on these vegetation types and their peculiarities. The palm swamps in the park have many more aquatic plants and more riparian species typical of the Pantanal (Pott et al., 2011) than those in the area of Rio Verde/Coxim. Worth mentioning is that the park had just being created, so there was no time yet for vegetation changes due to cattle exclusion. The seasonal regime of flood and dry, characteristic of the Pantanal, is probably why

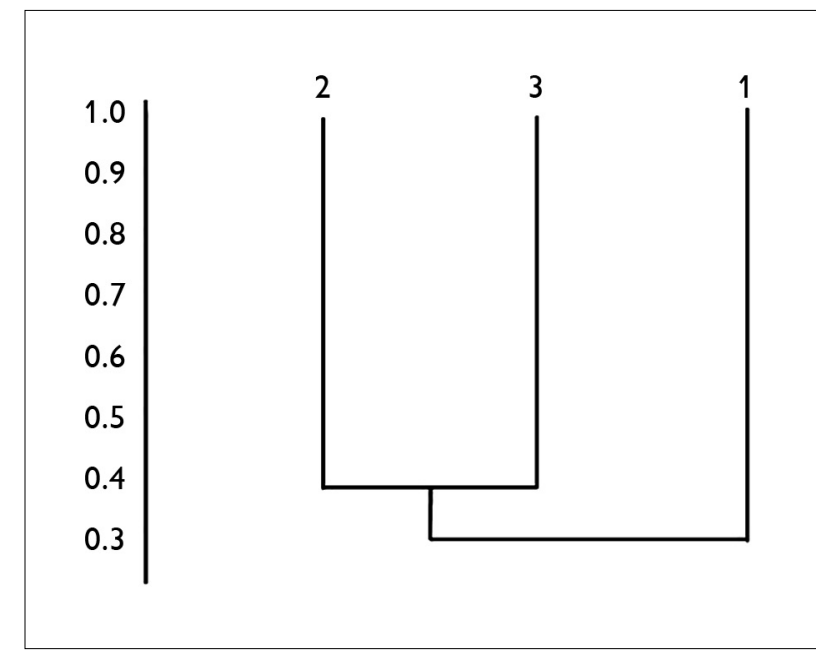

Figure 2. Bray-Curtis similarity based on plant species occurrences in veredas (1), Mauritia flexuosa palmlands of the Pantanal (2), and aquatic macrophytes of the Pantanal (3).

these palmlands in Aquidauana differ from permanently wet, true veredas.

Near the Aquidauana River there is moriche palm grove in its upper stretch inside the plain. Downstream from the junction with the Miranda River the moriche palm is no longer seen, replaced by another palm, Copernicia alba Morong ex Morong \& Britton, probably because of soil differences, due to sediments with higher $\mathrm{pH}$ from the Bodoquena plateau that is rich in limestone.

The probable reason for occurrence of palm swamps further north, near the pediment of the Maracaju highland, is surface water from small seasonal streams and the possibly rise of underground water from the upland. Such outflow is common in foothill veredas in the Cerrado region (Queiroz, 2009). Some palm swamps close to the outer border of the plain lack a defined stream, always present in true veredas, instead there is a flow of shallow seasonal flooding and prolonged waterlogging. That is the hydrological reason why they can be considered true veredas. In addition, the soil of the palmland on the plain is not as organic and waterlogged as in upland veredas, except the organic soil swamp in the Coxim area. 
In the Rio Negro Park some M. flexuosa groves occur by the river, in pure stands or mixed with riparian forest, and other areas were sampled away from the riverside, as monodominant savanna. Near the river, the palms looked healthy, with various ages, since there is open drainage, with subsurface water flow. However, farther from the river, we observed many dead moriche palms, particularly in the wettest areas, where young palms were scarce. Although M. flexuosa is considered flood tolerant, due to its pneumatophores (Pott \& Pott, 2004), it tends to have some mortality in years with excessive flooding. That seems paradoxal, but standing water in backwater-swamps may be unfavorable. We observed a similar population decline of the palm in the sub-region of Barão de Melgaço, attributed to the dam effect of a road. Furthermore, in spite of being fire tolerant, those aerial respiratory roots can be damaged under burned tall grasses in very dry years, as deduced from stems blackened by fire, and then the palm can die. Such deleterious effect of fire had already been observed in drained $M$. flexuosa swamps in the Ivinhema watershed (Pottet al., 2014). The interaction of flood with fire has been shown to occur in riparian forests of the Pantanal (Arruda et al., 2016). In spots with fallen palms there is succession to vines, known gap colonizers, e.g., Ipomoea rubens Choisy and Merremia umbellata (L.) Hallierf. We did not see signs of wildfire on the riverside palms, probably because of little flammable grass in the dense shrubby understorey.

The palmlands near the plain edge in the Rio Verde/ Coxim site show similarity with upland veredas, e.g., nine species of Utricularia, many Poaceae, Cyperaceae, and Melastomataceae, whereas the areas in the park have a flora composed of aquatic and seasonally flooded grassland plants and riparian scrub from the Pantanal, as had already been suggested by Pott et al. (2011). Thus, the eastern areas share only $1 / 3$ of the flora with the Rio Negro Park and are a transition to upland veredas.

We found a rare and endemic plant as a first record in the Pantanal, Polygala grazielae, only known from three previous collections by Gert Hatschbach, who discovered it along streams in the Rio Verde upland (CRIA, 2017).
The eastern border is the most cleared part of the Pantanal (Pott \& Silva, 2015), leaving the M. flexuosa stripes and patches isolated, as the surrounding woodland (cerradão) has been converted into Urochloa spp. pastures. The lowland palm swamps are also used for cattle ranching, though grazing has recently stopped inside the park. The organic soil vereda in Coxim is too boggy for cattle, so it is still conserved. The Rio Verde areas are for most part invaded by Urochloa humidicola (Rendle) Morrone \& Zuloaga, and in one area the exotic grass was even planted, at least leaving the palms alive, but we did not observe seedlings. This point (Córrego Pindaivão) has seasonal ponds that were reported as veredas by Moreira (2015). Nonetheless, both the palm swamps and veredas should be preserved for their spatial diversity and uniqueness in the unique Pantanal.

\section{CONCLUSIONS}

We conclude that Mauritia flexuosa swamps of the Rio Negro Park are not true veredas, because most plant species are associated with the flooding grasslands of the Pantanal wetland, whereas the Rio Verde/Coxim areas can be considered true veredas, for sharing species with the flora of veredas of the upper basin and exhibiting some exclusive species do not occur elsewhere on the plain. Palm swamps and veredas in the Pantanal should receive the same legal protection as the veredas in the upland Cerrado.

\section{ACKNOWLEDGEMENTS}

The authors thank the Conselho Nacional de Desenvolvimento Científico e Tecnológico (CNPq) (Project n. 477675/2011-5, and scholarship to SNM); the Coordenação de Aperfeiçoamento de Pessoal de Nível Superior (CAPES) (Visiting Professorship grant to AP); the Programa de Pós-Graduação em Biologia Vegetal and the CGMS UFMS Herbarium; the Programa de Pós-Graduação em Biologia Vegetal UFMG, UEMS Campus Coxim; the Instituto de Meio Ambiente de Mato Grosso do Sul; biologist João Fabri, for help in the field; and Filipe Soares de Souza, M.Sc. in Botany, for the map. 


\section{REFERENCES}

ARRUDA, W. S., J. OLDELAND, A. C. PARANHOS FILHO, A. POTT, N. L. CUNHA, I. H. ISHI \& G. A. DAMASCENO-JUNIOR, 2016. Inundation and fire shape the structure of riparian forests in the Pantanal, Brazil. PLoS ONE 11(6): e0156825. DOI: <https:// doi.org/10.1371/journal.pone.0156825>.

BRASIL, 2002. Resolução n 303, de 20 de março de 1992. Dispõe sobre parâmetros, definições e limites de Áreas de Preservação Permanente. Diário Oficial da União, 13 maio 2002. Disponível em: <http://www.mma.gov.br/port/conama/res/res02/res30302. html >. Accessed on: October 27, 2017.

CARVALHO, P. G. S., 1991. As veredas e sua importância no domínio dos cerrados. Informe Agropecuário 15(168): 47-54.

CENTRO DE REFERÊNCIA E INFORMAÇÃO AMBIENTAL (CRIA), 2017. Specieslink. Avaiable at: <http://www.splink.org. br/index>. Accessed on: April 29, 2017.

FILGUEIRAS, T. S., A. L. BROCHADO, P. E. NOGUEIRA \& G. F. GUALA, 1994. Caminhamento: um método expedito para levantamentos florísticos qualitativos. Caderno de Geociências (12): 39-43.

MOREIRA, S. N., 2015. Flora, distribuição e estrutura da vegetação das áreas úmidas de uma região savânica brasileira: implicações para a conservação da biodiversidade: 1-131. Tese (Doutorado em Biologia Vegetal) - Universidade Federal de Minas Gerais, Belo Horizonte. Avaiable at: < http://hdl.handle.net/1843/ BUBD-A35JZ6 > . Accessed on: April 14, 2017.

MOREIRA, S. N., P. V. EISENLOHR, A. POTT, V. J. POTT \& A. T. OLIVEIRA-FILHO, 2015. Similar vegetation structure in protected and non-protected wetlands in Central Brazil: conservation significance. Environmental Conservation 42: 1-7.

OLIVEIRA, G. C., G. M. ARAÚJO \& A. A. A. BARBOSA, 2009. Florística e zonação de espécies vegetais em veredas no Triângulo Mineiro, Brasil. Rodriguésia 60(4): 1077-1085.

OLIVEIRA-FILHO, A. T. \& F. R. MARTINS, 1986. Distribuição, caracterização e composição florística das formações vegetais da região da Salgadeira, na Chapada do Guimarães (MT). Revista Brasileira de Botânica 9: 207-223.
POTT, V. J. \& A. POTT, 2000. Plantas aquáticas do Pantanal: 1-404. EMBRAPA, Brasília.

POTT, V. J. \& A. POTT, 2004. Buriti - Mauritia flexuosa. Fauna e Flora do Cerrado, out. 2004. Avaiable at: <http://cloud.cnpgc. embrapa.br/faunaeflora/plantas-uteis/buriti-mauritia-flexuosa>. Accessed on: May 6, 2017.

POTT, V. J., A. POTT, L. C. P. LIMA, A. N. MOREIRA \& A. K. OLIVEIRA, 2011. Aquatic macrophyte diversity of the Pantanal wetland and upper basin. Brazilian Journal of Biology 71(1): 255-263. DOI: <http://dx.doi.org/10.1590/S1519-69842011000200004>.

POTT, V. J., F. A. FERREIRA, A. C. V. ARANTES \& A. POTT, 2012. How many species of aquatic plants are there in the Brazilian Pantanal wetland? Anais do Congresso Brasileiro de Áreas Úmidas 1: 280-281.

POTT, A., J. N. V. SILVA \& E. L. GOMES, 2014. Características da Bacia Hidrográfica do Rio Ivinhema. Revista GeoPantanal 9(16): 109-124.

POTT, A. \& J. S. V. SILVA, 2015. Terrestrial and aquatic vegetation diversity of the Pantanal wetland. In: I. BERGIER \& M. ASSINE (Ed.): Dynamics of the Pantanal Wetland in South America: 111-131. Springer (The Handbook of Environmental Chemistry, v. 37), Cham. DOI: <https://doi.org/10.1007/698_2015_352>.

QUEIROZ, M. L., 2009. Nascentes, veredas, áreas úmidas: revisão conceitual e metodologia de caracterização e determinação: estudo de caso na Estação Ecológica de Águas Emendadas - Distrito Federal: 1-104. Dissertação (Mestrado em Geociências Aplicadas) Universidade de Brasília, Brasília.

SILVA, M. P., R. A. MAURO, G. MOURÃO \& M. COUTINHO, 2000. Distribuição e quantificação de classes de vegetação do Pantanal através de levantamento aéreo. Revista Brasileira de Botânica 23(2): 143-152. DOI: < http://dx.doi.org/10.1590/S010084042000000200004>. 
Appendix. Phanerogamic flora of Mauritia flexuosa palm swamps in the Pantanal wetland. Site 1 = Aquidauna and Site 2 = Rio Verde/ Coxim. Moreira et al. (2015) shows the common species between areas with and without M. flexuosa. Voucher collectors: SNM = Suzana Neves Moreira; VJP = Vali Joana Pott; AP = Arnildo Pott; $\mathrm{P}=$ present; $*$ = exotic species.

\begin{tabular}{|c|c|c|c|c|c|}
\hline Family & Species & Site 1 & Site 2 & $\begin{array}{l}\text { Exclusive } \\
\text { to site } 2\end{array}$ & $\begin{array}{l}\text { Moreira et al. } \\
\text { (2015) }\end{array}$ \\
\hline \multirow{4}{*}{ Acanthaceae } & Hygrophila costata Nees & VJP 8894 & - & - & - \\
\hline & Justicia laevilinguis (Nees) Lindau & VJP 8889 & SNM 1563 & - & - \\
\hline & Staurogyne diantheroides Lindau & $\mathrm{P}$ & SNM 1543 & - & - \\
\hline & Stenandrium diphyllum Nees & - & SNM 869 & - & - \\
\hline \multirow{8}{*}{ Alismataceae } & Echinodorus glaucus Rataj & VJP 8887 & $\mathrm{P}$ & - & - \\
\hline & E. longipetalus Michele & - & SNM 1565 & - & $x$ \\
\hline & E. paniculatus Michele & VJP 8225 & - & - & - \\
\hline & E. scaber Rataj & VJP 8226 & - & - & - \\
\hline & Helanthium tenellum (Mart.) Britton & VJP 8224 & SNM 818 & - & - \\
\hline & Limnocharis laforestii Duchass. & - & SNM 831 & - & - \\
\hline & Sagittaria guayanensis Kunth & $\mathrm{P}$ & AP 4475 & - & $x$ \\
\hline & S. rhombifolia Cham. & VJP 8223 & VJP 17371 & - & - \\
\hline Anacardiaceae & Tapirira guianensis Aubl. & - & $\mathrm{P}$ & - & $x$ \\
\hline \multirow{2}{*}{ Amaranthaceae } & Gomphrena elegans Mart. & VJP 8222 & - & - & - \\
\hline & Iresine macrophylla R.E. Fr. & AP 13435 & - & - & - \\
\hline \multirow{4}{*}{ Annonaceae } & Annona dioica Mart. & - & $P$ & - & - \\
\hline & Unonopsis guatterioides (A. DC.) R.E. Fr. & AP 13460 & - & - & - \\
\hline & Xylopia aromatica (Lam.) Mart. & & AP 2584 & & \\
\hline & X. emarginata (Lam.) Mart. & - & $\mathrm{P}$ & $x$ & $x$ \\
\hline \multirow{5}{*}{ Apocynaceae } & Funastrum clausum (Jacq.) Schltr. & $\mathrm{P}$ & - & - & - \\
\hline & Mandevilla rugosa (Benth.) Woodson & - & SNM 836 & $x$ & $x$ \\
\hline & Rhabdadenia madida (Vell.) Miers & VJP 8901 & - & - & - \\
\hline & $\begin{array}{c}\text { Tabernaemontana siphilitica (L.f.) } \\
\text { Leeuwenb. }\end{array}$ & AP 13447 & - & - & - \\
\hline & $\begin{array}{c}\text { Tassadia berteroana (Spreng.) W.D. } \\
\text { Stevens }\end{array}$ & $P$ & $P$ & - & - \\
\hline Aquifoliaceae & Ilex affinis Gardner & - & SNM 1563 & $x$ & $x$ \\
\hline \multirow{5}{*}{ Araceae } & Dieffenbachia aglaonaematifolia Engl. & $\mathrm{P}$ & - & - & - \\
\hline & Pistia stratiotes L. & VJP 8276 & - & - & - \\
\hline & Urospatha sagittifolia (Rudge) Schott & - & $\mathrm{P}$ & - & $x$ \\
\hline & $\begin{array}{c}\text { Xanthosoma striatipes (Kunth \& Bouché) } \\
\text { Madison }\end{array}$ & $P$ & $P$ & - & $x$ \\
\hline & Wolffiella lingulata (Hegelm.) Hegelm. & VJP 8888 & - & - & - \\
\hline Araliaceae & Hydrocotyle pusilla A. Rich. & - & SNM 1562 & $x$ & - \\
\hline
\end{tabular}


Appendix.

(Continue)

\begin{tabular}{|c|c|c|c|c|c|}
\hline Family & Species & Site 1 & Site 2 & $\begin{array}{l}\text { Exclusive } \\
\text { to site } 2\end{array}$ & $\begin{array}{l}\text { Moreira et al. } \\
\quad(2015)\end{array}$ \\
\hline \multirow{3}{*}{ Arecaceae } & Attalea phalerata Mart. ex Spreng. & $\mathrm{P}$ & - & - & - \\
\hline & Bactris glaucescens Druce & $\mathrm{P}$ & - & - & - \\
\hline & Mauritia flexuosa L. f. & VJP 8904 & SNM 952 & - & $x$ \\
\hline \multirow{13}{*}{ Asteraceae } & $\begin{array}{c}\text { Acilepidopsis echitifolia (Mart. ex DC.) } \mathrm{H} \text {. } \\
\text { Rob. }\end{array}$ & - & VJP 2077 & $x$ & $x$ \\
\hline & Aspilia latissima Malme & $P$ & - & - & - \\
\hline & Baccharis glutinosa Pers. & AP 2146 & $\mathrm{P}$ & - & $x$ \\
\hline & Barrosoa candolleana (Hook. \& Arn.) & VJP 8263 & - & - & $x$ \\
\hline & $\begin{array}{l}\text { Chromolaena lilacina (Hieron.) R.M. King } \\
\text { \& H. Rob. }\end{array}$ & - & SNM 848 & $x$ & - \\
\hline & Elephantopus palustris Gardner & - & SNM 832 & $x$ & $x$ \\
\hline & Erechtites hieracifolius (L.) Rafin & - & SNM 1566 & - & - \\
\hline & Erigeron tweediei Hook. \& Arn. & - & $\mathrm{P}$ & $x$ & $x$ \\
\hline & Mikania micrantha Kunth & VJP 3240 & - & - & - \\
\hline & M. stenophylla W.C. Holes & & SNM 870 & - & - \\
\hline & $\begin{array}{c}\text { Praxelis clematidea (Griseb.) R.M. King \& } \\
\text { H. Rob. }\end{array}$ & VJP 8251 & - & - & - \\
\hline & $\begin{array}{l}\text { Urolepis hecatantha (DC.) K.M. King \& } \\
\text { H. Rob. }\end{array}$ & $\mathrm{P}$ & - & - & - \\
\hline & Vernonanthura brasiliana (L.) H. Rob. & $\mathrm{P}$ & $\mathrm{P}$ & - & - \\
\hline \multirow{2}{*}{ Bignoniaceae } & Handroanthus heptaphyllus Mattos & $\mathrm{P}$ & - & - & - \\
\hline & Tabebuia insignis (Miq.) Sandwith & - & AP 6574 & - & $x$ \\
\hline Boraginaceae & $\begin{array}{c}\text { Euploca filiformis (Lehm.) J.I.M. Melo \& } \\
\text { Semir }\end{array}$ & AP 5765 & - & - & - \\
\hline \multirow[t]{2}{*}{ Burmanniaceae } & $\begin{array}{c}\text { Burmannia capitata (Walter ex J.F. Gmel.) } \\
\text { Mart. }\end{array}$ & - & AP 2198 & - & - \\
\hline & B. flava Mart. & - & AP 2172 & & \\
\hline Burseraceae & Protium heptaphyllum (Mart.) Marchand & VJP 8262 & - & - & - \\
\hline Bromeliaceae & Bromelia balansae Mez & $P$ & - & - & \\
\hline Cabombaceae & Cabomba furcata Schult. \& Schult. f. & VJP 8233 & SNM 1546 & - & - \\
\hline \multirow{2}{*}{ Campanulaceae } & Lobelia aquatica Cham. & - & SNM 1528 & - & $x$ \\
\hline & L. anceps L.f. & - & $\mathrm{P}$ & $x$ & \\
\hline Cannaceae & Canna glauca L. & VJP 8274 & - & - & - \\
\hline Capparidaceae & $\begin{array}{c}\text { Cleoserrata paludosa (Willd. ex Eichler) } \\
\text { Iltis }\end{array}$ & AP 14185 & - & - & - \\
\hline Celastraceae & Hippocratea volubilis L. & AP 13437 & $P$ & - & - \\
\hline
\end{tabular}


Appendix.

(Continue)

\begin{tabular}{|c|c|c|c|c|c|}
\hline Family & Species & Site 1 & Site 2 & $\begin{array}{l}\text { Exclusive } \\
\text { to site } 2\end{array}$ & $\begin{array}{l}\text { Moreira et al. } \\
\qquad(2015)\end{array}$ \\
\hline Celastraceae & Salacia elliptica (Mart. ex Schult.) G. Don & AP 5431 & - & - & - \\
\hline \multirow{2}{*}{ Chrysobalanaceae } & Hirtella gracilipes (Hook. f.) Prance & - & AP 6511 & - & - \\
\hline & Licania parvifolia Huber & $P$ & - & - & - \\
\hline \multirow{2}{*}{ Clusiaceae } & Calophyllum brasiliense Cambess. & AP 14181 & $P$ & - & - \\
\hline & Garcinia brasiliensis Mart. & AP 14461 & - & - & - \\
\hline \multirow{2}{*}{ Combretaceae } & Combretum lanceolatum Pohl & $P$ & - & - & - \\
\hline & C. rotundifolium Aubl. & VJP 3690 & - & - & - \\
\hline \multirow{2}{*}{ Commelinaceae } & Commelina schomburgkiana Klotzsch & VJP 3712 & - & - & - \\
\hline & Murdannia gardneri (Seub.) G. Brückn. & VJP 1451 & $P$ & - & - \\
\hline \multirow{5}{*}{ Convolvulaceae } & Aniseia martinicensis (Jacq.) Choisy & VJP 8896 & SNM 852 & - & - \\
\hline & Ipomoea alba L. & $P$ & - & - & - \\
\hline & I. chiliantha Hallier f. & $P$ & - & - & - \\
\hline & Ipomoea rubens Choisy & $P$ & - & - & - \\
\hline & Merremia umbellata (L.) Hallier f. & AP 5812 & - & - & - \\
\hline Costaceae & Costus arabicus L. & $P$ & - & - & - \\
\hline \multirow{2}{*}{ Cucurbitaceae } & Melothria candolleana Cogn. & AP 13434 & & - & - \\
\hline & Psiguria ternata (M.J. Roem.) C. Jeffrey & P & AP 2414 & - & \\
\hline \multirow{18}{*}{ Cyperaceae } & $\begin{array}{c}\text { Ascolepis brasiliensis (Kunth) Benth. ex } \\
\text { C.B. Clarcke }\end{array}$ & AP 5898 & $P$ & - & \\
\hline & Bulbostylis of. fluviatilis Kral \& Davidse & - & SNM 789 & - & - \\
\hline & Cyperus compressus L. & AP 5337 & - & & \\
\hline & C. cornelii-ostenii Kük. & AP 5834 & - & & \\
\hline & C. giganteus Vahl & VJP 8246 & - & - & \\
\hline & C. haspan L. & AP 5892 & $P$ & - & $x$ \\
\hline & C. odoratus L. & VJP 3709 & SNM 874 & - & - \\
\hline & C. prolixus Kunth & - & SNM 828 & - & - \\
\hline & C. surinamensis Rottb. & VJP 3708 & $P$ & - & - \\
\hline & Eleocharis acutangula (Roxb.) Steud. & $P$ & SNM 799 & - & $x$ \\
\hline & E. elegans (Kunth) Roem. \& Schult. & VJP 8228 & $P$ & - & - \\
\hline & E. filiculmis Kunth & - & SNM 795 & - & - \\
\hline & E. interstincta (Vahl) Roem. \& Schult. & VJP 8648 & $\mathrm{P}$ & - & - \\
\hline & E. minima Kunth & VJP 8229 & SNM 812 & - & - \\
\hline & E. mutata (L.) Roem. \& Schult. & $P$ & $P$ & - & - \\
\hline & E. plicarhachis (Griseb.) Svenson & VJP 7893 & $P$ & - & - \\
\hline & Eleocharis sp. & - & SNM 784 & - & - \\
\hline & Fimbristylis dichotoma (L.) Vahl & VJP 8612 & SNM 844 & - & - \\
\hline
\end{tabular}


Appendix.

(Continue)

\begin{tabular}{|c|c|c|c|c|c|}
\hline Family & Species & Site 1 & Site 2 & $\begin{array}{l}\text { Exclusive } \\
\text { to site } 2\end{array}$ & $\begin{array}{c}\text { Moreira et al. } \\
\text { (2015) }\end{array}$ \\
\hline \multirow{19}{*}{ Cyperaceae } & Fuirena umbellata Rottb. & VJP 8269 & $P$ & - & $x$ \\
\hline & Killinga brevifolia Rottb. & AP 5822 & AP 2559 & - & - \\
\hline & Lipocarpha humboldtiana Nees & AP 5864 & AP 2375 & - & - \\
\hline & Oxycaryum cubense (Poepp. \& Kunth) Lye & VJP 5726 & - & - & - \\
\hline & Rhynchospora brevirostris Griseb. & - & SNM 813 & - & - \\
\hline & R. corymbosa (L.) Britton & VJP 8219 & - & - & - \\
\hline & R. emaciata (Nees) Boeckeler & - & AP 2567 & $x$ & $x$ \\
\hline & R. globosa (Kunth) Roem. \& Schult. & - & $P$ & - & - \\
\hline & R. hirta (Nees) Boeckeler & & AP 2485 & & \\
\hline & R. tenuis Link & VJP 8261 & - & - & - \\
\hline & R. trispicata (Nees) Schrad. ex Steud. & AP 5768 & SNM 819 & - & - \\
\hline & R. velutina (Kunth) Boeckeler & VJP 4117 & SNM 823 & - & - \\
\hline & Scleria gaertneri Raddi & VJP 8679 & SNM 825 & & \\
\hline & S. hirtella Sw. & $\mathrm{P}$ & $\mathrm{P}$ & & \\
\hline & S. leptostachya Kunth & & AP 2484 & & \\
\hline & S. lithosperma (L.) Sw. & - & $\mathrm{P}$ & - & - \\
\hline & S. macrophylla J. Presl \& C. Presl & - & SNM 838 & $x$ & $x$ \\
\hline & S. microcarpa Nees ex Kunth & - & $\mathrm{P}$ & - & - \\
\hline & S. secans (L.) Urb. & $\mathrm{P}$ & - & - & - \\
\hline \multirow{3}{*}{ Dilleniaceae } & Curatella americana L. & - & VJP 2418 & - & - \\
\hline & Davilla nitida (Vahl) Kubitszi & - & SNM 834 & $x$ & $x$ \\
\hline & Doliocarpus dentatus (Aubl.) Standl. & $\mathrm{P}$ & - & - & - \\
\hline Dioscoreaceae & Dioscorea hassleriana Chodat & - & $\mathrm{P}$ & - & - \\
\hline \multirow{2}{*}{ Droseraceae } & Drosera communis A. St.-Hil. & - & SNM 1549 & $x$ & - \\
\hline & D. sessilifolia A. St.-Hil. & - & AP 2199 & - & - \\
\hline \multirow{5}{*}{ Eriocaulaceae } & Eriocaulon sellowianum Kunth & - & $\mathrm{P}$ & $x$ & - \\
\hline & Paepalanthus chiquitensis Herzog & AP 13465 & AP 2232 & & \\
\hline & Philodice hoffmannseggii Mart. & - & SNM 816 & - & - \\
\hline & Syngonanthus caulescens (Poir.) Ruhland & - & $P$ & - & - \\
\hline & S. gracilis (Bong.) Ruhland & VJP 3679 & SNM 804 & - & $x$ \\
\hline Erythroxylaceae & Erythroxylum anguifugum Mart. & AP 13459 & AP 1300 & - & - \\
\hline \multirow{4}{*}{ Euphorbiaceae } & Alchornea castaneifolia (Willd.) A. Juss. & $\mathrm{P}$ & - & - & - \\
\hline & A. discolor Poepp. & AP 13453 & AP 2414 & - & - \\
\hline & Caperonia castaneifolia (L.) A. St.-Hil. & AP 5747 & AP 2573 & - & - \\
\hline & Sapium obovatum Klotzsch ex Müll. Arg. & AP 5407 & AP 2565 & - & $x$ \\
\hline Fabaceae & Aeschynomene fluminensis Vell. & VJP 8238 & $P$ & - & - \\
\hline
\end{tabular}


Appendix.

(Continue)

\begin{tabular}{|c|c|c|c|c|c|}
\hline Family & Species & Site 1 & Site 2 & $\begin{array}{l}\text { Exclusive } \\
\text { to site } 2\end{array}$ & $\begin{array}{l}\text { Moreira et al. } \\
\qquad(2015)\end{array}$ \\
\hline \multirow{21}{*}{ Fabaceae } & A. sensitiva Sw. & VJP 8237 & SNM 821 & - & - \\
\hline & $\begin{array}{c}\text { Albizia inundata (Mart.) Barneby \& J.W. } \\
\text { Grimes }\end{array}$ & $P$ & - & - & - \\
\hline & $\begin{array}{c}\text { Ancistrotropis peduncularis (Kunth) A. } \\
\text { Delgado }\end{array}$ & - & AP 16357 & - & - \\
\hline & Andira inermis (W. Wright) DC. & AP 13876 & - & - & - \\
\hline & $\begin{array}{c}\text { Calopogonium caeruleum (Benth.) C. } \\
\text { Wright }\end{array}$ & AP 14187 & - & - & - \\
\hline & C. mucunoides Desv. & - & $P$ & - & - \\
\hline & Cassia grandis L.f. & $P$ & - & - & - \\
\hline & Clitoria falcata Lam. & $P$ & $P$ & - & - \\
\hline & Desmodium barbatum Benth. & $P$ & SNM 876 & - & - \\
\hline & D. cajanifolium (Kunth) DC. & - & AP 16355 & $x$ & $x$ \\
\hline & D. scorpiurus (Sw.) Desv. & - & SNM 859 & - & - \\
\hline & Erythrina fusca Lourt. & AP 13438 & - & - & - \\
\hline & Inga vera subsp. affinis (DC.) T.D. Penn. & AP 14176 & - & - & - \\
\hline & Machaerium amplum Benth. & AP 4773 & - & - & - \\
\hline & Mimosa pellita Humb. \& Bonpl. ex Willd. & AP 5835 & - & - & - \\
\hline & Pterocarpus michelii Britton & AP 13445 & - & - & - \\
\hline & $\begin{array}{c}\text { Senna aculeata (Pohl ex Benth.) H.S. } \\
\text { Irwin \& Barneby }\end{array}$ & $P$ & - & - & - \\
\hline & S. alata (L.) Roxb. & $P$ & - & - & - \\
\hline & S. pendula (Willd.) H.S. Irwin \& Barneby & AP 14189 & - & - & - \\
\hline & $\begin{array}{c}\text { Stylosanthes acuminata M.B. Ferreira \& } \\
\text { Souza Costa }\end{array}$ & - & SNM 867 & - & - \\
\hline & Vigna longifolia (Benth.) Verdc. & AP 14178 & SNM 863 & - & - \\
\hline \multirow{3}{*}{ Gentianaceae } & Chelonanthus alatus (Aubl.) Pulle & - & SNM 947 & $x$ & $x$ \\
\hline & Coutoubea ramosa Aubl. & AP 13215 & - & - & - \\
\hline & Schultesia heterophylla Miq. & AP 5850 & AP 2210 & & \\
\hline \multirow{2}{*}{ Heliconiaceae } & Heliconia marginata (Griggs) Pittier & VJP 8116 & $P$ & - & - \\
\hline & H. psittacorum L. f. & $P$ & $P$ & - & - \\
\hline \multirow{3}{*}{ Hydrocharitaceae } & Egeria najas Planch. & VJP 8234 & - & - & - \\
\hline & $\begin{array}{l}\text { Limnobium laevigatum (Humb. \& Bonpl. } \\
\text { ex Willd.) Heine }\end{array}$ & VJP 8880 & - & - & - \\
\hline & Najas microcarpa K. Schum. & VJP 8245 & - & - & - \\
\hline Hydroleaceae & Hydrolea spinosa L. & VJP 8877 & SNM 862 & - & - \\
\hline
\end{tabular}


Appendix.

(Continue)

\begin{tabular}{|c|c|c|c|c|c|}
\hline Family & Species & Site 1 & Site 2 & $\begin{array}{l}\text { Exclusive } \\
\text { to site } 2\end{array}$ & $\begin{array}{l}\text { Moreira et al. } \\
\text { (2015) }\end{array}$ \\
\hline \multirow{10}{*}{ Lamiaceae } & $\begin{array}{c}\text { Cantinoa carpinifolia (Benth.) Harley \& } \\
\text { J.B.F. Pastore }\end{array}$ & - & SNM 854 & $x$ & - \\
\hline & Hyptis balansae Briq. & - & SNM 1501 & $x$ & $x$ \\
\hline & H. brevipes Poit. & VJP 8265 & $\mathrm{P}$ & - & - \\
\hline & H. conferta Pohl ex Benth. & & SNM 814 & $x$ & \\
\hline & H. crenata Pohl ex Benth. & - & SNM 944 & - & - \\
\hline & H. dumetorum Morong & - & SNM 781 & - & $x$ \\
\hline & H. lorentziana O. Hoffm. & VJP 8239 & SNM 861 & - & - \\
\hline & H. lappacea Benth. & AP 5797 & - & - & - \\
\hline & H. sinuata Pohl ex Benth. & - & SNM 969 & - & $x$ \\
\hline & Vitex cymosa Bertero & $\mathrm{P}$ & - & - & - \\
\hline \multirow{4}{*}{ Lauraceae } & Cassytha filiformis L. & - & SNM 841 & - & - \\
\hline & Nectandra amazonum Nees & $\mathrm{P}$ & - & - & - \\
\hline & N. gardneri Meisn. & - & SNM 833 & $x$ & $x$ \\
\hline & Ocotea diospyrifolia (Meisn.) Mez & $\mathrm{P}$ & - & - & - \\
\hline \multirow{16}{*}{ Lentibulariaceae } & Genlisea repens Benj. & - & SNM 1571 & $x$ & $x$ \\
\hline & Utricularia breviscapa Wright ex Griseb. & $P$ & $P$ & - & - \\
\hline & U. erectiflora A. St.-Hil. \& Girard & - & SNM 1502 & $x$ & $x$ \\
\hline & U. foliosa L. & AP 5806 & - & - & - \\
\hline & U. gibba L. & VJP 8232 & SNM 803 & - & - \\
\hline & U. hydrocarpa Vahl & - & SNM 1504 & - & - \\
\hline & U. Iloydii Merl ex F. Lloyd & - & SNM 1557 & $x$ & - \\
\hline & U. nana A. St.-Hil. \& Girard & - & $\mathrm{P}$ & - & - \\
\hline & U. nervosa G. Weber ex Benj. & - & SNM 1503 & $x$ & $x$ \\
\hline & U. nigrescens Sylvén & - & $P$ & $x$ & - \\
\hline & U. olivacea Wright ex Griseb. & - & $\mathrm{P}$ & $x$ & - \\
\hline & U. pusilla Vahl & - & SNM 947 & $x$ & - \\
\hline & U. simulans Pilg. & - & SNM 800 & - & - \\
\hline & U. subulata L. & - & SNM 943 & $x$ & - \\
\hline & U. trichophylla Spruce ex Oliv. & - & SNM 946 & $x$ & - \\
\hline & U. tricolor A. St.-Hil. & - & SNM 1505 & $x$ & $x$ \\
\hline \multirow{2}{*}{ Limnocharitaceae } & Hydrocleys nymphoides (Willd.) Buchenau & VJP 8879 & VJP 8879 & - & - \\
\hline & H. parviflora Seub. & & AP 6189 & & \\
\hline \multirow{3}{*}{ Lythraceae } & Cuphea odonellii Lourteig & - & SNM 791 & - & - \\
\hline & C. retrorsicapilla Koehne & AP 7623 & $\mathrm{P}$ & - & - \\
\hline & Rotala mexicana Cham. \& Schltdl. & - & SNM 871 & - & - \\
\hline
\end{tabular}


Appendix.

(Continue)

\begin{tabular}{|c|c|c|c|c|c|}
\hline Family & Species & Site 1 & Site 2 & $\begin{array}{l}\text { Exclusive } \\
\text { to site } 2\end{array}$ & $\begin{array}{c}\text { Moreira et al. } \\
\qquad(2015)\end{array}$ \\
\hline Malpighiaceae & Byrsonima cydonifolia A. Juss. & $P$ & AP 2370 & - & - \\
\hline \multirow{10}{*}{ Malvaceae } & Byttneria genistella Triana \& Olanch. & VJP 8635 & AP 2489 & & \\
\hline & B. palustris Cristóbal & - & SNM 1508 & - & - \\
\hline & B. rhamnifolia Benth. & $P$ & - & - & - \\
\hline & Corchorus argutus L. & - & SNM 868 & - & - \\
\hline & Hibiscus furcellatus Desr. & VJP 8221 & - & - & - \\
\hline & Hibiscus sororius L. f. & VJP 8252 & SNM 847 & - & - \\
\hline & Melochia simplex A. St.-Hil. & VJP 8902 & SNM 866 & - & - \\
\hline & M. villosa (Mill.) Fawc. \& Rendle & $P$ & AP 2583 & - & - \\
\hline & Pavonia laetevirens R.E. Fr. & $P$ & - & - & - \\
\hline & Peltaea riedelii (Gürcke) Standl. & AP 5400 & AP 7658 & - & - \\
\hline Marantaceae & Thalia geniculata L. & AP 5816 & SNM 864 & - & - \\
\hline \multirow{2}{*}{ Mayacaceae } & Mayaca fluviatilis Aubl. & $P$ & SNM 824 & - & - \\
\hline & M. sellowiana Kunth & - & SNM 1510 & $x$ & - \\
\hline \multirow{17}{*}{ Melastomataceae } & Acisanthera alsinaefilia Triana & AP 5873 & $P$ & - & - \\
\hline & A. divaricata Cogn. & - & SNM 882 & - & - \\
\hline & A. limnobios (DC.) Triana & $P$ & SNM 794 & - & - \\
\hline & Clidemia hirta (L.) G. Don & - & $P$ & - & - \\
\hline & Desmoscelis villosa (Aubl.) Naudin & - & SNM 844 & - & - \\
\hline & Macairea radula (Bonpl.) DC. & - & SNM 1516 & $x$ & - \\
\hline & Miconia albicans (Sw.) Triana & - & SNM 796 & - & - \\
\hline & M. prasina (Sw.) DC. & AP 5312 & SNM 822 & - & - \\
\hline & Poteranthera pusilla Bong. & & AP 2224 & $x$ & \\
\hline & Pterolepis trichotoma (Rottb.) Cogn & - & SNM 1514 & $x$ & - \\
\hline & Rhynchanthera grandiflora (Aubl.) DC. & AP 8013 & SNM 858 & - & - \\
\hline & R. novemnervia DC. & VJP 8250 & SNM 830 & - & - \\
\hline & R. ursina Naudin & - & SNM 1518 & $x$ & - \\
\hline & Siphanthera arenaria (DC.) Cogn. & - & SNM 856 & - & - \\
\hline & S. foliosa (Naudin) Wurdack & - & SNM 1515 & $x$ & - \\
\hline & Tibouchina gracilis (Bonpl.) Cogn. & AP 13466 & SNM 1220 & - & - \\
\hline & Trembleya phlogiformis DC. & - & SNM 780 & $x$ & - \\
\hline \multirow{2}{*}{ Meliaceae } & Trichilia pallida Sw. & AP 13432 & - & - & - \\
\hline & T. stellato-tomentosa Kuntze & $\mathrm{P}$ & - & - & - \\
\hline \multirow{2}{*}{ Menispermaceae } & Cissampelos pareira L. & VJP 3649 & AP 2570 & - & - \\
\hline & Odontocarya tamoides (DC.) Miers & AP 5339 & SNM 873 & - & - \\
\hline Menyanthaceae & Nymphoides indica (L.) Kuntze & - & $P$ & - & - \\
\hline
\end{tabular}


Appendix.

(Continue)

\begin{tabular}{|c|c|c|c|c|c|}
\hline Family & Species & Site 1 & Site 2 & $\begin{array}{l}\text { Exclusive } \\
\text { to site } 2\end{array}$ & $\begin{array}{c}\text { Moreira et al. } \\
\text { (2015) }\end{array}$ \\
\hline Molluginaceae & Mollugo verticillata $\mathrm{L}$. & AP 5391 & SNM 840 & - & - \\
\hline \multirow{3}{*}{ Moraceae } & Ficus adhatodifolia Schott ex Spreng. & $\mathrm{P}$ & $\mathrm{P}$ & - & - \\
\hline & F. lutschnatiana (Miq.) Miq. & AP 13448 & - & - & - \\
\hline & F. pertusa L.f. & AP 13451 & AP 2431 & - & - \\
\hline Myristicaceae & Virola sebifera Aubl. & - & SNM 786 & $x$ & - \\
\hline Myrsinaceae & Myrsine umbellata Mart. & - & $P$ & $x$ & - \\
\hline \multirow{3}{*}{ Myrtaceae } & Calyptranthes lucida Mart. ex DC. & AP 13446 & - & - & - \\
\hline & Eugenia florida DC. & AP 5310 & $\mathrm{P}$ & - & - \\
\hline & Psidium guineense DC. & $P$ & $P$ & - & - \\
\hline \multirow{2}{*}{ Nymphaeaceae } & Nymphaea amazonum Mart. \& Zucc. & VJP 8235 & VJP 2957 & - & - \\
\hline & N. gardneriana Planch. & $P$ & $P$ & - & - \\
\hline \multirow{4}{*}{ Ochnaceae } & Ouratea castaneifolia (DC.) Engl. & AP 14188 & - & - & - \\
\hline & Sauvagesia erecta $\mathrm{L}$. & VJP 8260 & $P$ & - & - \\
\hline & S. racemosa A. St.-Hil. & - & SNM 1520 & $x$ & $x$ \\
\hline & S. tenella Lam. & & AP 2222 & & \\
\hline \multirow{10}{*}{ Onagraceae } & Ludwigia affinis (DC.) O. Hara & $P$ & - & - & - \\
\hline & L. elegans (Cambess.) H. Hara & VJP 8217 & - & - & - \\
\hline & L. grandiflora (Michx.) Zardini & VJP 8884 & - & - & - \\
\hline & L. helminthorhiza (Mart.) H. Hara & AP 5729 & - & - & - \\
\hline & Ludwigia inclinata (L. f.) P.H. Raven & $\mathrm{P}$ & $\mathrm{P}$ & - & - \\
\hline & L. leptocarpa (Nutt.) H. Hara & VJP 8242 & - & - & - \\
\hline & L. nervosa (Poir.) O. Hara & VJP 8244 & SNM 1522 & - & - \\
\hline & L. octovalvis (Jacq.) P.H. Raven & VJP 8866 & $P$ & - & - \\
\hline & L. sedoides (Humb. \& Bonpl.) H. Hara & VJP 8254 & - & - & - \\
\hline & L. tomentosa (Cambess.) ○. Hara & VJP 8873 & SNM 843 & - & - \\
\hline Orchidaceae & Cyrtopodium paludicolum Hoehne & - & SNM 1559 & $x$ & $x$ \\
\hline Orobanchaceae & Melasma stricta (Benth.) Hassl. & - & SNM 1523 & - & - \\
\hline Passifloraceae & Passiflora misera Kunth & VJP 8241 & - & - & - \\
\hline \multirow{2}{*}{ Phyllanthaceae } & Hieronyma alchornoides Allemão & - & SNM 785 & $x$ & - \\
\hline & Phyllanthus stipulatus Webster & VJP 8595 & SNM 811 & - & - \\
\hline \multirow{2}{*}{ Piperaceae } & Piper fuligineum Kunth & - & $\mathrm{P}$ & $x$ & $x$ \\
\hline & P. macedoi Yunck. & - & VJP 11442 & - & - \\
\hline \multirow{4}{*}{ Plantaginaceae } & Angelonia salicarifolia Bonpl. & VJP 3752 & - & - & - \\
\hline & Bacopa arenaria (J.A. Sm.) Edwall & $P$ & AP 6193 & - & - \\
\hline & B. australis V.C. Souza & VJP 8231 & - & - & - \\
\hline & B. myriophylloides (Benth.) Wettst. & VJP8258 & SNM 805 & - & - \\
\hline
\end{tabular}


Appendix.

(Continue)

\begin{tabular}{|c|c|c|c|c|c|}
\hline Family & Species & Site 1 & Site 2 & $\begin{array}{l}\text { Exclusive } \\
\text { to site } 2\end{array}$ & $\begin{array}{l}\text { Moreira et al. } \\
\text { (2015) }\end{array}$ \\
\hline \multirow{5}{*}{ Plantaginaceae } & B. monnierioides (Cham.) B.L. Rob. & VJP 8259 & SNM 1526 & - & - \\
\hline & B. reflexa (Benth.) Edwall & - & SNM 1561 & - & - \\
\hline & B. salzmannii (Benth.) Wettst. ex Edwall & VJP 8230 & SNM 1525 & - & - \\
\hline & B. stricta (Schrad.) Wettst. ex Edwall & AP 5365 & SNM 839 & - & - \\
\hline & Scoparia dulcis L. & AP 13470 & AP 2286 & - & - \\
\hline \multirow{28}{*}{ Poaceae } & Acroceras zizanioides (Kunth) Dandy & AP 5807 & AP 5907 & - & - \\
\hline & Andropogon bicornis L. & VJP 8895 & AP 2556 & - & - \\
\hline & A. hypogynus Hack. & $P$ & AP 2367 & - & - \\
\hline & A. leucostachyus Kunth & - & SNM 798 & - & - \\
\hline & A. virgatus Desv. & $P$ & AP 2219 & - & - \\
\hline & Anthaenantia lanata (Kunth.) Benth. & - & AP 2357 & - & - \\
\hline & $\begin{array}{c}\text { Arundinella hispida (Humb, \& Bonpl. ex } \\
\text { Willd.) Kuntze }\end{array}$ & $P$ & - & - & - \\
\hline & Axonopus leptostachyus (Flüggé) Hitchc. & AP 5837 & AP 1104 & - & - \\
\hline & A. siccus (Nees) Kuhlm. & - & SNM 808 & - & $x$ \\
\hline & Coleataenia stenodes (Griseb.) Soreng & $P$ & AP 1125 & - & - \\
\hline & Cyphonanthus discrepans (Döll) & - & AP 2909 & & \\
\hline & Digitaria ciliaris (Retz.) Koeler & - & SNM 855 & - & - \\
\hline & Echinochloa crus-galli (L.) P. Beauv. & AP 5769 & - & & \\
\hline & Echinolaena gracilis Swallen & - & SNM 820 & - & - \\
\hline & Eragrostis rufescens Schrad. ex Schult. & - & SNM 885 & - & - \\
\hline & Eriochrysis warmingiana (Hack.) Kuhlmann & - & $P$ & $x$ & $x$ \\
\hline & $\begin{array}{c}\text { Gymnopogon burchelii (Munro ex Döll) } \\
\text { Munro }\end{array}$ & - & $P$ & $x$ & - \\
\hline & G. fastigiatus Nees & - & AP 2220 & & \\
\hline & Hymenachne amplexicaulis (Rudge) Nees & AP 5351 & $P$ & - & - \\
\hline & H. donacifolia (Raddi) Chase & VJP 8683 & - & - & - \\
\hline & H. pernambucencis (Spreng.) Zuloaga & AP 5778 & - & - & - \\
\hline & *Hyparrhenia rufa (Nees) Stapf & - & SNM 845 & - & - \\
\hline & Imperata tenuis Hack. & VJP 2730 & AP 2557 & - & - \\
\hline & Leersia hexandra Sw. & $P$ & $\mathrm{P}$ & - & - \\
\hline & Loudetia flammida (Trin.) C.E. Hubb. & - & SNM 846 & - & - \\
\hline & $\begin{array}{l}\text { Louisiella elephantipes (Nees ex Trin.) } \\
\text { Zuloaga }\end{array}$ & $P$ & - & - & - \\
\hline & Luziola bahiensis (Steud.) Hitchc. & - & $P$ & & \\
\hline & L. fragilis Swallen & VJP 1702 & AP 6185 & - & - \\
\hline
\end{tabular}


Appendix.

(Continue)

\begin{tabular}{|c|c|c|c|c|c|}
\hline Family & Species & Site 1 & Site 2 & $\begin{array}{l}\text { Exclusive } \\
\text { to site } 2\end{array}$ & $\begin{array}{l}\text { Moreira et al. } \\
\quad(2015)\end{array}$ \\
\hline \multirow{32}{*}{ Poaceae } & Mnesithea aurita (Steud.) de Koning \& Sosef & $\mathrm{P}$ & SNM 829 & - & - \\
\hline & $\begin{array}{l}\text { Oedochloa procurrens (Nees ex Trin.) C. } \\
\text { Silva \& R.P. Oliveira (IChnanthus) }\end{array}$ & Valls 10394 & AP 2497 & & \\
\hline & Oplismenus hirtellus (L.) P. Beauv. & AP 5401 & - & - & - \\
\hline & Otachyrium versicolor (Döll) Henrard & - & SNM 851 & - & - \\
\hline & Panicum caaguazuense Henrard & - & $P$ & $x$ & - \\
\hline & P. dichotomiflorum Michx. & AP 5751 & VJP 8605 & & \\
\hline & *P. repens L. & $\mathrm{P}$ & SNM 842 & - & - \\
\hline & P. spectabile Nees ex Trin. & - & SNM 820 & & - \\
\hline & P. trichanhum Nees & VJP 3659 & - & & \\
\hline & Paspalum atratum Swallen & - & $P$ & - & - \\
\hline & P. ellipticum Döll & - & SNM 1577 & - & - \\
\hline & P. fasciculatum Willd. ex Flüggé & $P$ & - & - & - \\
\hline & P. lineare Trin. & - & AP 2354 & & \\
\hline & P. maculosum Trin. & - & SNM 1535 & $x$ & - \\
\hline & $\begin{array}{c}\text { P. morichalense Davidse, Zuloaga \& } \\
\text { Filgueiras }\end{array}$ & - & SNM 853 & - & - \\
\hline & P. multicaule Poir. & - & $\mathrm{P}$ & & \\
\hline & P. plicatulum Michx. & $\mathrm{P}$ & AP 2359 & - & - \\
\hline & P. repens Bergius & Valls 10414 & & - & - \\
\hline & P. stellatum Humb. \& Bonpl. ex Flüggé & - & SNM 783 & - & - \\
\hline & Reimarochloa acuta (Flüggé) Hitchc. & AP 5347 & AP 2474 & - & - \\
\hline & Rhytachne rottboellioides Desv. ex Ham. & - & AP1123 & & \\
\hline & Rugoloa hylaeica (Mez) Zuloaga & $\mathrm{P}$ & - & - & - \\
\hline & R. polygonata (Schrad.) Zuloaga & VJP 8893 & - & - & - \\
\hline & Saccharum asperum (Nees) Steud. & - & SNM 827 & $x$ & $x$ \\
\hline & S. villosum Steud. & - & SNM 826 & $x$ & $x$ \\
\hline & $\begin{array}{c}\text { Sacciolepis angustissima (Hochst. ex } \\
\text { Steud.) Kuhlm. }\end{array}$ & & AP 2211 & & \\
\hline & S. myuros (Lam.) Chase & $\mathrm{P}$ & SNM 801 & - & - \\
\hline & S. vilvoides (Trin.) Chase & - & $\mathrm{P}$ & - & - \\
\hline & Schizachyrium gracilipes (Hack.) A. Camus & - & SNM 790 & $x$ & - \\
\hline & S. microstachyum Roseng. & - & $\mathrm{P}$ & - & - \\
\hline & Setaria parviflora Kerguelen & AP 5794 & AP 2364 & - & - \\
\hline & $\begin{array}{c}\text { Stephostachys mertensii (Roth) Zuloaga \& } \\
\text { Morrone }\end{array}$ & $P$ & AP 6660 & - & - \\
\hline
\end{tabular}


Appendix.

(Continue)

\begin{tabular}{|c|c|c|c|c|c|}
\hline Family & Species & Site 1 & Site 2 & $\begin{array}{l}\text { Exclusive } \\
\text { to site } 2\end{array}$ & $\begin{array}{l}\text { Moreira et al. } \\
\qquad(2015)\end{array}$ \\
\hline \multirow{4}{*}{ Poaceae } & Sorghastrum setosum (Griseb.) Hitchc. & AP 5893 & AP 2494 & - & - \\
\hline & Steinchisma laxum (Sw.) Zuloaga & AP 5568 & AP 2473 & - & - \\
\hline & $\begin{array}{l}\text { Trichanthecium parvifolium (Lam.) Zuloaga } \\
\text { \& Morrone }\end{array}$ & - & SNM 802 & - & - \\
\hline & $\begin{array}{c}\text { *Urochloa humidicola (Rendle) Morrone } \\
\text { \& Zuloaga }\end{array}$ & P & $P$ & - & - \\
\hline \multirow{5}{*}{ Polygalaceae } & Polygala appendiculata Vell. & - & SNM 787 & - & - \\
\hline & P. grazielae Marques & - & SNM 787 & $x$ & - \\
\hline & P. tenuis DC. & $P$ & AP 2277 & & \\
\hline & P. timoutoides Chodat & - & SNM 1540 & - & - \\
\hline & P. timoutou Aubl. & - & SNM 880 & - & - \\
\hline \multirow{5}{*}{ Polygonaceae } & Polygonum acuminatum Kunth & VJP 3662 & - & - & - \\
\hline & P. ferrugineum Wedd. & $P$ & - & - & - \\
\hline & P. hydropiperoides Michx. & VJP 8272 & - & - & - \\
\hline & P. punctatum Elliot & AP 3815 & SNM 857 & - & - \\
\hline & Triplaris americana L. & AP 13433 & - & - & - \\
\hline \multirow{6}{*}{ Pontederiaceae } & Eichhornia azurea (Sw.) Kunth & VJP 2915 & AP 6191 & - & - \\
\hline & E. crassipes (Mart.) Solms & VJP 2916 & - & - & - \\
\hline & Pontederia cordata L. & AP 5761 & $\mathrm{P}$ & & \\
\hline & P. parviflora Alexander & VJP 8227 & SNM 1511 & - & - \\
\hline & P. rotundifolia L. f. & VJP 8881 & - & - & - \\
\hline & P. subovata (Seub.) Lowden & VJP 8236 & - & - & - \\
\hline \multirow{9}{*}{ Rubiaceae } & Alibertia edulis (Rich.) A. Rich. & AP 6543 & $P$ & - & - \\
\hline & $\begin{array}{c}\text { Amaioua glomerulata (Lam. ex Poir.) } \\
\text { Delprete \& C. Persson }\end{array}$ & AP 13202 & - & - & - \\
\hline & Borreria latifolia (Aubl.) K. Schum. & - & SNM 884 & - & - \\
\hline & $\begin{array}{c}\text { B. pulchristipula (Bremek.) Bacigalupo \& } \\
\text { E.L. Cabral }\end{array}$ & - & $P$ & $x$ & $x$ \\
\hline & $\begin{array}{l}\text { Cephalanthus glabratus (Spreng.) K. } \\
\text { Schum. }\end{array}$ & AP 14180 & - & - & - \\
\hline & $\begin{array}{c}\text { Coccocypselum lanceolatum (Ruiz \& Pav.) } \\
\text { Pers. }\end{array}$ & - & SNM 1556 & $x$ & - \\
\hline & Diodia kuntzei K. Schum. & AP 5346 & AP 2236 & - & - \\
\hline & $\begin{array}{l}\text { D. radula (Willd. \& Hoffmanns. ex Roem. } \\
\qquad \text { \& Schult.) Cham. \& Schltdl. }\end{array}$ & - & $P$ & $x$ & - \\
\hline & Genipa americana L. & AP 5327 & - & - & - \\
\hline
\end{tabular}


Appendix.

\begin{tabular}{|c|c|c|c|c|c|}
\hline Family & Species & Site 1 & Site 2 & $\begin{array}{l}\text { Exclusive } \\
\text { to site } 2\end{array}$ & $\begin{array}{l}\text { Moreira et al. } \\
\quad(2015)\end{array}$ \\
\hline \multirow{4}{*}{ Rubiaceae } & Limnosipanea palustris (Seem.) Hook.f. & - & SNM 1509 & $x$ & - \\
\hline & Psychotria carthagenensis Jacq. & AP 5425 & SNM 1542 & - & - \\
\hline & Sabicea aspera Aubl. & AP 5786 & $\mathrm{P}$ & - & - \\
\hline & Sipanea biflora (L. f.) Cham. \& Schtdl. & AP 14186 & - & - & - \\
\hline Rutaceae & $\begin{array}{l}\text { Zanthoxylum rigidum Humb. \& Bonpl. ex } \\
\text { Willd. }\end{array}$ & $\mathrm{P}$ & - & - & - \\
\hline \multirow{2}{*}{ Salicaceae } & Banara arguta Briq. & AP 5375 & - & - & - \\
\hline & Cardiospermum halicacabum L. & AP 5745 & $\mathrm{P}$ & - & - \\
\hline Sapindaceae & Paullinia pinnata L. & AP 5783 & $P$ & - & - \\
\hline Smilacaceae & Smilax fluminensis Vell. & $\mathrm{P}$ & AP 16354 & - & - \\
\hline \multirow{3}{*}{ Solanaceae } & Schwenckia americana L. & - & SNM 815 & - & - \\
\hline & S. juncoides Chodat & - & SNM 815 & $x$ & - \\
\hline & Solanum nigrescens M. Martens \& Galeotti & VJP 8903 & - & - & - \\
\hline Typhaceae & Typha domingensis Pers. & VJP 8275 & - & - & - \\
\hline \multirow{2}{*}{ Urticaceae } & Cecropia pachystachya Trécul & $\mathrm{P}$ & $\mathrm{P}$ & - & - \\
\hline & Urera aurantiaca Wedd. & $\mathrm{P}$ & - & - & - \\
\hline \multirow{3}{*}{ Vitaceae } & Cissus erosa Rich. & AP 5736 & AP 2517 & - & - \\
\hline & C. spinosa Cambess. & VJP 8247 & $\mathrm{P}$ & - & - \\
\hline & C. verticillata (L.) Nicolson \& C.E. Jarvis & $\mathrm{P}$ & - & - & - \\
\hline Vochysiaceae & Vochysia divergens Pohl & $P$ & $P$ & - & - \\
\hline \multirow{4}{*}{ Xyridaceae } & Xyris jupicai L.C. Rich. & VJP 8264 & $\mathrm{P}$ & - & - \\
\hline & X. macrocephala Vahl & - & SNM 807 & $x$ & \\
\hline & $X$. savanensis Miq. & $\mathrm{P}$ & SNM 817 & - & - \\
\hline & X. schizachne Mart. & - & SNM 1544 & $x$ & - \\
\hline
\end{tabular}

